\title{
Covariates of amikacin disposition in a large pediatric oncology cohort
}

Isabelle Dewandel ${ }^{1}$, Karel Allegaert ${ }^{2,3,4}$, Marleen Renard ${ }^{5}$, Annouschka Laenen ${ }^{6}$, Anne Smits 2,7 *

${ }^{1}$ Department of Pediatrics, University Hospitals Leuven, Herestraat 49, 3000 Leuven, Belgium;

2 Department of Development and Regeneration, KU Leuven, Herestraat 49, 3000 Leuven, Belgium; ${ }^{3}$ Department of Pharmaceutical and Pharmacological Sciences, KU Leuven, Herestraat 49, 3000 Leuven, Belgium; ${ }^{4}$ Department of Clinical Pharmacy, Erasmus MC, Postbus 2040, 3000 GA Rotterdam, The Netherlands; ${ }^{5}$ Department of Pediatric hematooncology, University Hospitals Leuven, Herestraat 49, 3000 Leuven, Belgium; 6 Leuven Biostatistics and Statistical Bioinformatics Centre (L-Biostat), KU Leuven, Kapucijnenvoer 35 blok d - bus 7001, 3000 Leuven, Belgium; ${ }^{7}$ Neonatal intensive care unit, University Hospitals Leuven, Herestraat 49, 3000 Leuven, Belgium.

ORCID IDs: Karel Allegaert 0000-0001-9921-5105; Annouschka Laenen 0000-0002-1371442x; Anne Smits 0000-0002-0710-6698

Funding: The study has not been funded. The research activities of Anne Smits are supported by the Clinical Research and Education Council of the University Hospitals Leuven.

* Correspondence: Anne Smits, MD, PhD

Neonatal intensive care unit, University Hospitals Leuven

Herestraat 49, 3000 Leuven, Belgium

Phone: +32 16 343565, Fax: +32 16343209 ,

Email: anne.smits@uzleuven.be

Word count abstract:

Word count main text (introduction - discussion):

Number of figures:

Number of tables:

Number of Supplementary tables:
217 words

2992 words

3 Figures

5 Tables

2 Supplementary tables 


\section{What is known about this subject}

1. Aminoglycoside antibiotics are frequently used in children with febrile neutropenia.

2. Physiologic changes during development are responsible for age-related differences in amikacin pharmacokinetics (PK) in children.

3. Amikacin PK in children differs between disease groups (e.g. burns versus oncology diagnoses).

\section{What this study adds}

1. We explored amikacin PK in pediatric oncology patients, including within-patient changes across the treatment episodes.

2. Maturational covariates and creatinemia determined amikacin clearance and volume of distribution in a large cohort of pediatric oncology patients, while positive blood culture, oncology diagnosis and nephrotoxic chemotherapy were not retained as significant covariates.

3. The combination of clinical and PK data is useful to feed dosing software programs in the future, to improve drug exposure in special pediatric populations. 


\section{Abstract}

Objective: Amikacin pharmacokinetics (PK) in children displays large variability due to maturational and disease-related covariates. The objective was to explore amikacin PK in a large pediatric oncology cohort, taking into account within-patient changes.

Methods: Clinical data and amikacin therapeutic drug monitoring (TDM) observations were collected retrospectively from children with oncology diagnosis receiving amikacin during febrile neutropenia. Individual amikacin PK parameters were calculated using a onecompartment model with instantaneous input and first-order output. To explore covariates of clearance $(\mathrm{Cl})$ and volume of distribution $(\mathrm{Vd})$, linear mixed models were used, modelling a random effect for patient to account for clustering due to repeated measurements.

Results: Based on 188 amikacin treatment episodes in 114 patients, median (interquartile range) amikacin $\mathrm{Cl}$ was $1.37(1.05 ; 2.46) \mathrm{L} / \mathrm{h}$ and $\mathrm{Vd} 7.98(5.66 ; 12.73) \mathrm{L}$. Height and creatinemia were significant covariates for $\mathrm{Cl}$ (marginal $\mathrm{R}^{2} 71.1 \%$ ), while weight, height and creatinemia determined $\mathrm{Vd}$ (marginal $\mathrm{R}^{2}$ 59.5\%).

Conclusions: We described extensive variability of amikacin PK in a large cohort of pediatric oncology patients, including within-patient changes across treatment episodes. Maturational covariates and creatinemia determined amikacin $\mathrm{Cl}$ and $\mathrm{Vd}$, while primary non-maturational covariates were not significant. Our observations, based on combined clinical and PK data in children with oncology diagnoses, can be useful to feed dosing software programs to improve drug exposure in special populations.

Key words: amikacin - pharmacokinetics - febrile neutropenia - children 
Running title: Amikacin disposition in pediatric oncology patients 


\section{Introduction}

Aminoglycosides are one of the oldest antibiotic classes, who have played a major role in antimicrobial therapy since their discovery in the 1940s. It are hydrophilic compounds displaying a concentration dependent efficacy, while the trough level is associated with toxicity. Within this class, amikacin has the broadest in vitro and in vivo activity against most aerobic gram-negative bacteria. 'Once daily-dosing' or 'extended interval dosing' has been proven to be superior in neonates, children and adults, compared to multiple daily dosing $[1,2,3]$. This specific approach can be applied due to concentration-dependent bactericidal activity with therapeutic peak concentration; a post-antibiotic effect which allows continued efficacy even when serum concentrations are below expected minimal inhibitory concentration (MIC); a decreased risk of adaptive resistance; and reduced renal accumulation due to achievement of low trough concentrations $[4,5,6]$. Physiologic changes during development are responsible for age-related differences in amikacin disposition in children. A decrease in the body-water content with age affects the apparent volume of distribution $(\mathrm{Vd})$, while age-dependent renal maturation determines amikacin clearance $(\mathrm{Cl})$. Consequently, these maturational covariates (like age, weight, renal function...) result in large inter- and intra-individual amikacin pharmacokinetic $(\mathrm{PK})$ variability in children requiring optimized dosing regimens $[2,7,8]$. In addition, non-maturational covariates (like disease, co-medication, treatment) can further contribute to this PK variability, especially in high-risk pediatric populations (e.g. critically ill, febrile neutropenia, poly-pharmacy) [9]. The impact of disease condition on amikacin disposition has recently been documented in children with burn injuries compared to oncology patients [10]. Liu et al demonstrated that if other covariates (i.e. maturational covariates and creatinine clearance) remain the same, children with burn injuries exhibited higher central volume $(+17 \%)$ and $\mathrm{Cl}(+55 \%)$ compared to oncology patients [10]. Besides these between group differences in amikacin PK parameters, variability in amikacin disposition within the 
pediatric oncology subgroup also remains high. In this study we aim to explore which covariates mainly determine amikacin PK in a large pediatric oncology group, taking into account 1) both maturational and non-maturational covariates, and 2) within-patient alterations of these covariates.

\section{Methods}

\section{Study population}

Clinical data and amikacin therapeutic drug monitoring (TDM) observations were collected retrospectively from patient files of children with an oncologic diagnosis, admitted to the University Hospitals Leuven, Belgium, between January 2010 and June 2013, due to febrile neutropenia. This dataset in part overlays with Liu et al [10]. However, instead of only the first febrile neutropenia episode, now all episodes of individual patients are included, and data of 2 additional patients could be retrieved. Amikacin, co-administered with ceftazidim, was the empirical treatment for febrile neutropenia. The ethical board of the hospital approved the study (internal number S55690).

The following covariates were collected: sex, oncologic diagnosis (leukemia or solid tumor), induction phase chemotherapy (yes/no), previous administration of nephrotoxic chemotherapeutic drugs (i.e. carboplatin, cisplatin or ifosfamide), age (months), height $(\mathrm{cm})$, weight $(\mathrm{kg})$, creatinemia $(\mathrm{mg} / \mathrm{dL})$, albuminemia $(\mathrm{g} / \mathrm{L})$, c-reactive protein $(\mathrm{CRP}, \mathrm{mg} / \mathrm{L})$, blood culture at the start of amikacin therapy (positive/negative) and in case of positive blood culture, type of organism. Biochemical parameters were included if available in 24 hours before or after the moment of TDM. Blood cultures were observed at the start of each treatment episode. 


\section{Drug administration}

Amikacin was infused intravenously over 30 minutes. All patients were treated with the same dosing regimen i.e. $20 \mathrm{mg} / \mathrm{kg}$ once daily. Early exposure was evaluated, using the first trough $\left(\mathrm{C}_{\min }\right.$, measured prior to the second dose $)$ and peak observation $\left(\mathrm{C}_{\max 30}\right.$, concentration 30 minutes after finalization of the second amikacin administration, or $1 \mathrm{~h}$ after start of this administration) of each amikacin treatment episode.

\section{Amikacin assay}

Amikacin plasma concentrations were measured with fluorescence polarization immunoassay (Abbott TDx, Abbott Park, IL, USA). The assay was linear from 0.8 to $50.0 \mathrm{mg} / \mathrm{L}$ with intraday and inter-day variability less than $7.6 \%$. This analytical method was used until 31 May 2012, after which a kinetic interaction of microparticles in solution (KIMS) immunoassay (Roche/Hitachi Cobas c systems, Roche Diagnostics GmbH, Mannheim, Germany) was used for amikacin quantification with a coefficient of variation less than 5\%. To avoid censoring of values below the lower limit of quantification (LLOQ $0.8 \mathrm{mg} / \mathrm{L}$ ), these concentrations were replaced by LLOQ/2 (i.e. $0.4 \mathrm{mg} / \mathrm{L}$ ) as suggested in literature [11].

\section{Biochemical assay}

Creatinine and albumin in plasma were measured on Roche Modular P before April 2012 and with Roche Cobas c702 from April 2012 (Roche Diagnostics, Mannheim, Germany). All creatinine measurements were isotope dilution mass spectrometry (IDMS) traceable. Creatinine was measured enzymatically in patients $<5$ years and based on modified Jaffé in patients $\geq 5$ years until September 2012. From October 2012, creatinine was measured enzymatically in all patients. Albumin was measured using bromocresol green. 


\section{Pharmacokinetic parameters}

Individual amikacin PK parameters were calculated based on $\mathrm{C}_{\min }$ and $\mathrm{C}_{\max }{ }^{\prime} 30$, using a onecompartment model with instantaneous input and first-order output. $\mathrm{C}_{\max }{ }^{\prime} 30$ (concentration 30 minutes after finalization of the first amikacin administration, or $1 \mathrm{~h}$ after start of this administration) was calculated as $C_{\max 30}-C_{\min }$. Logarithm ( $\left.\log \right)$ of $C_{\max }{ }^{3} 30$ and $C_{\min }$ were used to calculate the slope of the individual trendline (equation 1)

Slope $=\left(\log _{C \max 130}-\log _{C \min }\right) /\left(t_{C \max 130}-t_{C \min }\right)$

(equation 1)

with $t_{C \max 30}$ and $t_{C \min }$ the time $(\mathrm{h})$ corresponding to the respective concentrations at $1 \mathrm{~h}$ and $24 \mathrm{~h}$ after start of the first administration. Subsequently, the elimination rate constant $\left(\mathrm{K}_{\mathrm{E}}\right)$ and elimination half-life $\left(\mathrm{T}_{1 / 2}, \mathrm{~h}\right)$ could be determined using equation 2 and equation 3 respectively.

Slope $=-K_{E} / 2.303$

(equation 2)

$T_{1 / 2}=0.693 / K_{E}$

(equation 3)

$\mathrm{C}_{\max 0}$ could be derived from equation 4

$\log _{C \max 130}=\log _{C_{\max 0}}-[($ Ke.t $) / 2.303]$

(equation 4)

With $\mathrm{C}_{\max 0}$ plasma amikacin concentration immediately after intravenous administration and $\mathrm{t}$ $=1 \mathrm{~h}$ after start of administration. Equation 4 represents a straight line indicating that the semilogarithmic plot of $\log _{C}$ versus time is linear with $\log _{C \max 0}$ as $Y$-intercept. Based on the dose administered and $\mathrm{C}_{\max 0}$, volume of distribution $\left(\mathrm{V}_{\mathrm{d}}, \mathrm{L}\right)$, and subsequently Clearance $(\mathrm{Cl}, \mathrm{L} / \mathrm{h})$ can be calculated using equations 5 and 6.

$V_{d}=$ Dose $/ C_{\max 0}$ (equation 5)

$C l=V_{d} \cdot K_{E}$ (equation 6) 


\section{Statistical analyses}

Continuous data are reported as median and interquartile range (IQR) or mean (SD), categorical data as incidence and percentage. To explore the effect of covariates on $\mathrm{Cl}$ and $\mathrm{Vd}$, linear mixed models were used, modelling a random effect for patient to account for clustering due to repeated measurements. A backward selection procedure was applied for multivariable model construction, with a 5\% significance level for covariates to be removed from the model. Nonlinear (quadratic) trends for continuous covariates were considered in the model building, if significant in univariable analysis. Given the presence of repeated measurements in the data, a classical $\mathrm{R}^{2}$ value could not be determined. As an alternative, we estimated the marginal $\mathrm{R}^{2}$, proposed by Nakagawa and Schielzeth for such clustered data settings, providing the proportion of variance explained by the fixed factors [12]. Based on the multivariable model, predicted $\mathrm{Cl}$ and Vd can be obtained as the weighted sum of the covariate measurements, with the parameter estimates as weights. The effect estimate is considered as the change in $\mathrm{Cl}$ or $\mathrm{Vd}$ for the indicated increase of the predictor level.

Analyses have been performed using SAS software (version 9.4, SAS System for Windows) and MedCalc version 19.1.5 (MedCalc Software, Ostend, Belgium). A p-value <0.05 was considered statistically significant.

\section{Results}

Between January 2010 and June 2013, 780 amikacin TDM results were available. Observations with missing TDM, TDM after sampling error and TDM collected later in the treatment episode (i.e. follow-up TDM) were excluded. From this dataset, treatment episodes for which both the first peak and trough, as well as dosing were available, were selected. Observations in nononcologic cases were excluded. Finally, first peak and trough of 188 amikacin treatment episodes, collected in 114 patients were retained for further analysis. The flowchart of included 
observations is presented in Figure 1. In total, $n=38 / 114$ patients $(33 \%)$ had $\geq 2$ amikacin treatment episodes ( $n=16$ patients with 2 episodes, $n=16$ with $3, n=3$ with $4, n=1$ with $5, n=1$ with 6 and n=1 with 9 episodes), counting for 112/188 episodes. Overall 105 trough levels were $<$ LLOQ and replaced by $0.4 \mathrm{mg} / \mathrm{L}$.

\section{Descriptive statistics}

Median (IQR) age of the included treatment episodes was 80 (40.50-151.50) months, and median weight $21.15(14.30-45.45) \mathrm{kg}$. Table 1 summarizes the clinical and biochemical characteristics. Twenty-five blood cultures were positive, 153 were negative and 10 unknown. The top 3 of isolated pathogens were Staphylococcus epidermidis $(n=8)$, Escherichia coli $(n=5)$ and Staphylococcus hominis $(n=4)$.

\section{Pharmacokinetic parameters}

Median observed amikacin trough level $\left(\mathrm{C}_{\min }\right)$ was $0.40(0.40-1.15)$ and peak $\left(\mathrm{C}_{\max 30}\right) 48.65$ (38.40-60.60) mg/L. Median amikacin $\mathrm{Cl}$ was $1.37(1.05-2.46) \mathrm{L} / \mathrm{h}$ with half-life $\left(\mathrm{T}_{1 / 2}\right) 3.56$ (3.3-4.33) h, and Vd 7.98 (5.66-12.73) L. To explore the impact of (non-)maturational covariates on $\mathrm{Cl}$ and $\mathrm{Vd}$, first univariable analyses were performed. For $\mathrm{Cl}$, age, weight, height, creatinemia and CRP were significant in univariable analyses. Blood culture (positive vs negative) and oncologic diagnosis (solid tumor vs leukemia) were no significant covariates of amikacin $\mathrm{Cl}$ (Table 2). In a final multivariable model, height and creatinine remained significant covariates of $\mathrm{Cl}$, with a marginal $\mathrm{R}^{2}$ of $71.1 \%$ (Table 3a). In Table $\mathbf{3 b}$, the effects per covariate are presented. Due to the quadratic effect, the change in $\mathrm{Cl}$ for a $1 \mathrm{~cm}$ increase in height varies over the range of height. This means that the change in $\mathrm{Cl}$ is e.g. different when height goes from 100 to $101 \mathrm{~cm}$ compared to 120 to $121 \mathrm{~cm}$. The nonlinear trend for $\mathrm{Cl}$ by height and the linear trend for $\mathrm{Cl}$ by creatinine are presented in Figure $\mathbf{2 a}$ and $2 \mathbf{b}$ respectively. 
The same covariates, significant in univariate analysis for $\mathrm{Cl}$, were retained for $\mathrm{Vd}$ (Table 4). In a final multivariable model, weight, height and creatinine remained significant covariates of $\mathrm{Vd}$, with a marginal $\mathrm{R}^{2}$ of $59.5 \%$ (Table 5 ). The trends for $\mathrm{Vd}$ by height and creatinine are presented in Figure 3a and 3b respectively.

\section{Discussion}

In a large cohort of pediatric oncology patients, we explored covariates of amikacin PK. Height and creatinemia were significant covariates of $\mathrm{Cl}$, and weight, height and creatinemia determined amikacin $\mathrm{Vd}$. The strengths of this analysis are the extensive cohort, with availability of combined (non-)maturational clinical and PK data, and the fact that withinpatient changes are taken into account. Since 112/188 episodes originated from patients with $\geq 2$ amikacin courses, these observations count for a substantial part (almost 60\%) of the dataset. Amikacin is frequently used in critically ill children. Our analysis confirms the substantial remaining PK variability. As a consequence, it is recommended to continue TDM assessments in special populations. Recently, amikacin disposition in pediatric subgroups e.g. neonates with asphyxia and hypothermia [13], children with burns [14], cystic fibrosis [15] and cancer [16] have been reported. Implementation of PK covariates in optimized dosing regimens, can improve safe and effective amikacin therapy in these populations. The maturational covariate height was retained on $\mathrm{Cl}$ instead of weight, which is more often retained in earlier reports. In a recent review, Illamola et al summarized the most common significant determinants of amikacin PK in parametric population PK analyses in neonates and children [7]. In the included (non-oncology) studies ( $\mathrm{n}=8), 6$ involved neonates up to 3 months, 2 investigated children $>2$ years (burn/ non-burn group). Current BW (cBW) was a $\mathrm{Cl}$ covariate in 7/8 studies, followed by age-related covariates and predictors of glomerular filtration rate (GFR) as most important 
$\mathrm{Cl}$ covariates in the neonatal studies. In addition, $\mathrm{cBW}$ was founds as covariate for $\mathrm{Vd}$ in all studies. Also in a recent study of $n=67$ children with (suspected) Gram-negative infection cBW was included as only covariate for both $\mathrm{Cl}$ and $\mathrm{Vd}$ in an amikacin PK/PD model [17]. In our cohort, exclusively containing oncology children, height was a more powerful predictor of $\mathrm{Cl}$. This might be related to the fact that weight displays larger within-patient fluctuations in oncology patients, compared to height. To further explore this, weight and height data of the 38 cases with multiple treatment episodes were selected. Mean (SD) percentage (\%) difference between first and last value, as well as maximal difference versus first value for weight were 3.658 (11.179) and $3.866(11.488) \%$, while 0.868 (2.178) and 0.854 (2.273)\% for height, respectively. Furthermore, additional analyses of $\mathrm{Cl}$, first expressed by $\mathrm{L} / \mathrm{h} . \mathrm{BSA},(\mathrm{BSA}=$ body surface area) were performed. Both height and weight were competing variables for inclusion in this model, of which the model building procedure selected height, in addition to creatinine (Table S1). Multivariable analysis of $\mathrm{Cl}$, expressed in $\mathrm{L} / \mathrm{kg} / \mathrm{h}$ revealed only creatinine as significant covariate (Table S2). Overall, these additional explorations did not reveal new predictors. Since height did not substantially changed within an individual during the study, amikacin $\mathrm{Cl}$ variability in our cohort seems mainly attributed to variation in creatinemia. Mean (SD) \% difference between first and last value, and maximal difference versus first value for creatinine were $24.026(60.030)$ and $31.670(9.729) \%$ respectively, indicating large intrapatient creatinine alterations. Creatinemia is a 'mixed covariate', reflecting a combined effect of maturation and non-maturational factors affecting renal function like disease, co-medication or treatment modalities.

Blood culture, CRP, oncology diagnosis and nephrotoxic chemotherapy were also explored as specific non-maturational predictors. Although CRP was significant in univariate analysis of $\mathrm{Cl}$, CRP nor blood culture were retained in the final multivariate selection. Theoretically, antibiotic $\mathrm{Cl}$ during sepsis can increase or decrease depending on renal function [18]. In 
addition, increased $\mathrm{Vd}$ due to sepsis-induced third space losses, fluid resuscitation and/or altered protein binding affects Vd of hydrophilic compounds. This is even more pronounced in burn patients with sepsis [19]. Oncology diagnosis was not significant in our analysis. In contrast, in a dataset including 207 adults with hematological malignancies, cBW and renal function, but also acute myeloblastic leukemia (amikacin $\mathrm{Cl}+20 \%$ ) and hypoalbuminemia (amikacin $\mathrm{Vd}+30.4 \%$ ) were found as most important covariates of amikacin disposition [20].

Amikacin $\mathrm{Cl}$ in our study is slightly higher compared to general ward and ICU children [mean Cl 1.78 (median 1.37) vs $1.2 \mathrm{~L} / \mathrm{h}$ ] [17], and comparable to critically ill children with normal renal function [mean $\mathrm{Cl} 0.07$ (median 0.06) vs $0.068 \mathrm{~L} / \mathrm{h} / \mathrm{kg}$ ] respectively [21]. This is in line with findings in adults with hematological and solid malignancies, in whom increased aminoglycoside $\mathrm{Cl}$ and $\mathrm{Vd}$ are reported earlier [22, 23, 24, 25]. As summarized by Hartman et $a l$, critically ill children generally show a higher $\mathrm{Cl}$ and larger $\mathrm{Vd}$ for antibiotics than healthy children and critically ill adults, resulting in a failure to achieve target concentrations for many antibiotics, including amikacin [26]. In contrast to renal dysfunction, hemodynamic changes during critical illness may result in increased renal clearance, known as augmented renal clearance (ARC) [27]. This is interesting, since amikacin is eliminated completely by glomerular filtration. Higher $\mathrm{Cl}$ during critical illness (e.g. sepsis) is partly attributed to this phenomenon, with ARC reported in up to $67 \%$ of critically ill children [28]. In children with cancer, 'glomerular hyperfiltration' is a recently recognized, short-lived phenomenon, present in $>40 \%$ of patients at diagnosis and during initial chemotherapy cycles [29]. The mechanism is not completely understood, but seems related to tumor volume with subsequent cell breakdown [30]. In addition, a hypermetabolic state is hypothesized to contribute to this hyperfiltration pathophysiology in children with cancer [29]. This might explain increased $\mathrm{Cl}$ of hydrophilic compounds in febrile neutropenia. For vancomycin, Hirai et al documented that 
febrile neutropenia indirectly increased $\mathrm{Cl}$ in children, due to this elevated GFR [31]. Consequently, increased $\mathrm{Cl}$ warrants higher dosing to achieve predefined targets. In a previous analysis of our group, simulations for children with cancer indeed indicated that higher amikacin doses than currently used are needed to achieve optimal peak and trough concentrations [10].

Our study has limitations. First, it concerns a retrospective analysis which only explored 'early' amikacin exposure, using an individual PK approach. Second, a 1-compartment model was selected. Also for other aminoglycosides in pediatric neutropenic fever, individual PK calculations using a 1-compartment model have been used [32]. For amikacin, both 1- and 2compartment models have been published in neonates and/or children [7, 17]. The a priori selection of a 1-compartment approach, is a limitation based on the pragmatic study design as TDM (peak/trough) observations as collected during clinical care were used. With 2 TDM samples per treatment episode, it is not feasible to estimate 2- or multiple compartment models.

As a future perspective, pooling of available amikacin observations in pediatric oncology cohorts might help to better understand PK determinants, and to improve dosing in this population. In addition, further exploration of PD aspects e.g. adaptive resistance (AR) during an amikacin treatment episode using in vivo MIC results, is needed. Alhadab et al developed an amikacin population PK model of pediatric oncology patients (n=34) [16]. An AR(PD) model of Pseudomonas aeruginosa from available in vitro time-kill curve data was combined with the PK model. Simulations based on this PK-ARPD approach suggested twice-daily dosing (45 mg/kg BID) for safe and effective therapy, while limiting bacterial resistance for this pathogen [16]. This type of analysis is important since dynamic evolution of bacterial growth and killing need to be considered. Especially, since mortality of P.aeruginosa 
bacteremia in this population remains constant last 20-30 years [33]. Recently, 30-day mortality of $P$. aeruginosa bacteremia in children with neutropenic fever was $38.9 \%$, and in $36.1 \%$ of bacteremia a multi-drug resistance strain was identified [33]. However, susceptibility of the isolated strains for amikacin was $100 \%$, compared to $67.6 \%$ for piperacillin/tazobactam and $72.2 \%$ for meropenem. This illustrates the potential importance of aminoglycosides in neutropenic fever, usually as part of empiric combination therapy.

\section{Conclusion}

We described extensive variability of amikacin PK in a very large cohort of pediatric oncology patients with febrile neutropenia, taking into account within-patient changes across amikacin treatment episodes. Maturational covariates and creatinemia determined amikacin $\mathrm{Cl}$ and $\mathrm{Vd}$, while primary non-maturational covariates were not significant. Our observations, based on combined clinical and PK data in children with an oncology diagnosis, are useful to feed dosing software programs to improve drug exposure in special populations. To achieve this, multidisciplinary collaboration between clinicians, microbiologists, pharmacologists and pharmacists is important [34]. Besides exploration of PK covariates, integrated PK/PD analyses with prospective in vivo MIC data over time, are useful to gain additional knowledge in clinical evolution of amikacin PD and to further improve amikacin exposure. 


\section{Table and Figure legends}

Table 1: Clinical and biochemical characteristics of the included observations.

Table 2: Univariable analyses of (non-)maturational covariates of clearance $(\mathrm{Cl}, \mathrm{L} / \mathrm{h})$

Table 3: Table 3a presents the final multivariable model for clearance $(\mathrm{Cl}, \mathrm{L} / \mathrm{h})$ resulting from a backward selection procedure; i.e. the selected covariates with their parameter estimates and significance. Table $3 \mathrm{~b}$ presents the covariate effects with $95 \%$ confidence intervals. Given that height follows a nonlinear trend, its effect on outcome is not constant over time, hence the effect is estimated at 3 different heights (i.e. percentile p25-50-75 of the study population, presented as rounded values $100-120-150 \mathrm{~cm})$.

Table 4: Univariable analyses of (non-)maturational covariates of volume of distribution (Vd, L).

Table 5: The final multivariable model for volume of distribution $(\mathrm{Vd}, \mathrm{L})$ resulting from a backward selection procedure; i.e. the selected covariates with their parameter estimates and significance as well as the covariate effects with $95 \%$ confidence intervals.

Supplementary Table S1: The multivariable model for clearance (Cl, L/h.BSA) resulting from a backward selection procedure; i.e. the selected covariates with their parameter estimates and significance as well as the covariate effects with $95 \%$ confidence intervals (BSA= Body Surface Area).

Supplementary Table S2: a) presents the multivariable model for clearance $(\mathrm{Cl}, \mathrm{L} / \mathrm{kg} / \mathrm{h})$ resulting from a backward selection procedure; i.e. the selected covariates with their parameter estimates and significance. b) Presents the covariate effects with $95 \%$ confidence intervals. Given that creatinemia follows a nonlinear trend, its effect on outcome is not constant over time, hence the effect is estimated at 3 different creatinine values (i.e. percentile p25-50-75 of the study population, presented as rounded values $0.3-0.5-0.7 \mathrm{mg} / \mathrm{dL}$ ). 
Figure 1: Flowchart of included amikacin therapeutic drug monitoring (TDM) results and amikacin treatment episodes.

Figure 2: The nonlinear trend (+ 95\% confidence interval in dashed lines) for amikacin clearance $(\mathrm{Cl}, \mathrm{L} / \mathrm{h})$ by height, for median creatinine of the entire dataset, is presented in Figure 3a. The linear trend (+ 95\% CI in dashed lines) for $\mathrm{Cl}$ by creatinine, for median height of the entire dataset, is presented in Figure 3b. Within the multivariable model, $\mathrm{Cl}$ is expressed as $\mathrm{Cl}$ $(\mathrm{L} / \mathrm{h})=1.1968-0.0179 *$ height $+0.0002 *$ height $*$ height $-1.1032 *$ creatinine $($ with height in $\mathrm{cm}$, creatinine in $\mathrm{mg} / \mathrm{dL}$ ).

Figure 3: Graphs presenting the linear trends for volume of distribution (Vd, L) by height, for median creatinine and weight (Figure 5a), and by creatinine, for median height and weight (Figure 5b). Medians are calculated based on the entire dataset. In the multivariable model, $\mathrm{Vd}$ is expressed as $\mathrm{Vd}(\mathrm{L})=-3.5156+0.0936 *$ weight $+0.1041 *$ height $-7.0313 *$ creatinine (with weight in $\mathrm{kg}$, height in $\mathrm{cm}$, creatinine in $\mathrm{mg} / \mathrm{dL}$ ). 


\section{Acknowledgements}

The research activities of Anne Smits are supported by the Clinical Research and Education Council of the University Hospitals Leuven.

\section{Conflict of interest}

The authors have no conflict of interest to declare. 


\section{References}

[1] Contopoulos-Ioannidis DG, Giotis ND, Baliatsa DV, Ioannidis JP. Extended-interval aminoglycoside administration for children: a meta-analysis. Pediatrics. 2004; 114: e111-

118.https://www.ncbi.nlm.nih.gov/pubmed/15231982

[2] Smits A, Kulo A, van den Anker J, Allegaert K. The amikacin research program: a stepwise approach to validate dosing regimens in neonates. Expert Opin Drug Metab Toxicol. 2017; 13: 157-

166.https://www.ncbi.nlm.nih.gov/pubmed/27623706 https://www.tandfonline.com/doi/full/10.1080/17425255.2017.1234606

[3] Krivoy N, Postovsky S, Elhasid R, Ben Arush MW. Pharmacokinetic analysis of amikacin twice and single daily dosage in immunocompromised pediatric patients. Infection. 1998; 26: 396-

398.http://www.ncbi.nlm.nih.gov/pubmed/9861567

[4] Germovsek E, Barker CI, Sharland M. What do I need to know about aminoglycoside antibiotics? Arch. Dis. Child Educ. Pract. Ed. 2017; 102: 89-93.http://www.ncbi.nlm.nih.gov/pubmed/27506599

[5] Pagkalis S, Mantadakis E, Mavros MN, Ammari C, Falagas ME. Pharmacological considerations for the proper clinical use of aminoglycosides. Drugs. 2011; 71: 2277-

2294.http://www.ncbi.nlm.nih.gov/pubmed/22085385

[6] Knoderer CA, Nichols KR, Cox EG. Optimized antimicrobial dosing strategies: a survey of pediatric hospitals. Paediatr Drugs. 2014; 16: 523-529.https://www.ncbi.nlm.nih.gov/pubmed/25315265

[7] Illamola SM, Sherwin CM, van Hasselt JGC. Clinical Pharmacokinetics of Amikacin in Pediatric Patients: A Comprehensive Review of Population Pharmacokinetic Analyses. Clin Pharmacokinet. 2018; 57: 1217-1228.https://www.ncbi.nlm.nih.gov/pubmed/29572662

[8] Kearns GL, Abdel-Rahman SM, Alander SW, Blowey DL, Leeder JS, Kauffman RE. Developmental pharmacology--drug disposition, action, and therapy in infants and children. N Engl J Med. 2003; 349 : $1157-$ 1167.https://www.ncbi.nlm.nih.gov/pubmed/13679531 https://www.nejm.org/doi/pdf/10.1056/NEJMra035092

[9] Allegaert K, Simons SHP, Tibboel D, Krekels EH, Knibbe CA, van den Anker JN. Non-maturational covariates for dynamic systems pharmacology models in neonates, infants, and children: Filling the gaps beyond developmental pharmacology. Eur J Pharm Sci. 2017; 109S: S27-

S31.https://www.ncbi.nlm.nih.gov/pubmed/28506866

[10] Liu X, Smits A, Wang Y, Renard M, Wead S, Kagan RJ, Healy DP, De Cock P, Allegaert K, Sherwin CMT. Impact of Disease on Amikacin Pharmacokinetics and Dosing in Children. Ther Drug Monit. 2019; 41: 44-52.https://www.ncbi.nlm.nih.gov/pubmed/30299427

[11] Beal SL. Ways to fit a PK model with some data below the quantification limit. J Pharmacokinet. Pharmacodyn. 2001; 28: 481-504.http://www.ncbi.nlm.nih.gov/pubmed/11768292

[12] Nakagawa S, Schielzeth $H$. A general and simple method for obtaining R2 from generalized linear mixed-effects models. Methods Ecol Evol. 2013; 4: 133-142.<Go to ISI>://WOS:000314974800004

[13] Cristea S, Smits A, Kulo A, Knibbe CAJ, van Weissenbruch M, Krekels EHJ, Allegaert K. Amikacin Pharmacokinetics To Optimize Dosing in Neonates with Perinatal Asphyxia Treated with Hypothermia.

Antimicrob Agents Chemother. 2017; 61.https://www.ncbi.nlm.nih.gov/pubmed/28993332

https://www.ncbi.nlm.nih.gov/pmc/articles/PMC5700363/pdf/e01282-17.pdf

[14] Sherwin CM, Wead S, Stockmann C, Healy D, Spigarelli MG, Neely A, Kagan R. Amikacin population pharmacokinetics among paediatric burn patients. Burns. 2014; 40: 311-

318.http://www.ncbi.nlm.nih.gov/pubmed/23876785

[15] Caceres Guido P, Perez M, Halac A, Ferrari M, Ibarra M, Licciardone N, Castanos C, Gravina LP, Jimenez C, Garcia Bournissen F, Schaiquevich P. Population pharmacokinetics of amikacin in patients with pediatric cystic fibrosis. Pediatr Pulmonol. 2019; 54: 18011810.https://www.ncbi.nlm.nih.gov/pubmed/31402602

[16] Alhadab AA, Ahmed MA, Brundage RC. Amikacin Pharmacokinetic-Pharmacodynamic Analysis in Pediatric Cancer Patients. Antimicrob Agents Chemother. 2018;

62.https://www.ncbi.nlm.nih.gov/pubmed/29358293

[17] Alqahtani S, Abouelkheir M, Alsultan A, Elsharawy Y, Alkoraishi A, Osman R, Mansy W. Optimizing Amikacin Dosage in Pediatrics Based on Population Pharmacokinetic/Pharmacodynamic Modeling. Paediatr Drugs. 2018; 20: 265-272.https://www.ncbi.nlm.nih.gov/pubmed/29569124

[18] Varghese JM, Roberts JA, Lipman J. Antimicrobial pharmacokinetic and pharmacodynamic issues in the critically ill with severe sepsis and septic shock. Crit Care Clin. 2011; 27: 19-

34.https://www.ncbi.nlm.nih.gov/pubmed/21144984

[19] Yu T, Stockmann C, Healy DP, Olson J, Wead S, Neely AN, Kagan RJ, Spigarelli MG, Sherwin CM. Determination of Optimal Amikacin Dosing Regimens for Pediatric Patients With Burn Wound Sepsis. J. Burn Care Res. 2015; 36: e244-e252.http://www.ncbi.nlm.nih.gov/pubmed/25185930 
[20] Romano S, Fdez de Gatta MM, Calvo MV, Caballero D, Dominguez-Gil A, Lanao JM. Population pharmacokinetics of amikacin in patients with haematological malignancies. J. Antimicrob. Chemother. 1999; 44: 235-242.http://www.ncbi.nlm.nih.gov/pubmed/10473231

[21] Marik PE, Havlik I, Monteagudo FS, Lipman J. The pharmacokinetic of amikacin in critically ill adult and paediatric patients: comparison of once- versus twice-daily dosing regimens. J Antimicrob Chemother.

1991; 27 Suppl C: 81-89.https://www.ncbi.nlm.nih.gov/pubmed/1856148

[22] Higa GM, Murray WE. Alterations in aminoglycoside pharmacokinetics in patients with cancer. Clin Pharm. 1987; 6: 963-966.https://www.ncbi.nlm.nih.gov/pubmed/3427879

[23] Kaojarern S, Maoleekoonpairoj S, Atichartakarn V. Pharmacokinetics of amikacin in hematologic malignancies. Antimicrob Agents Chemother. 1989; 33: 1406-

1408.https://www.ncbi.nlm.nih.gov/pubmed/2802567

[24] Manny RP, Hutson PR. Aminoglycoside volume of distribution in hematology-oncology patients. Clin Pharm. 1986; 5: 629, 632.https://www.ncbi.nlm.nih.gov/pubmed/3742952

[25] Zeitany RG, El Saghir NS, Santhosh-Kumar CR, Sigmon MA. Increased aminoglycoside dosage requirements in hematologic malignancy. Antimicrob. Agents Chemother. 1990; 34: 702-

708.http://www.ncbi.nlm.nih.gov/pubmed/2360811

[26] Hartman SJF, Bruggemann RJ, Orriens L, Dia N, Schreuder MF, de Wildt SN. Pharmacokinetics and Target Attainment of Antibiotics in Critically Ill Children: A Systematic Review of Current Literature. Clin Pharmacokinet. 2019.https://www.ncbi.nlm.nih.gov/pubmed/31432468

[27] Dhont E, Van Der Heggen T, De Jaeger A, Vande Walle J, De Paepe P, De Cock PA. Augmented renal clearance in pediatric intensive care: are we undertreating our sickest patients? Pediatr Nephrol. 2020; 35: 25-

39.https://www.ncbi.nlm.nih.gov/pubmed/30374606

[28] Van Der Heggen T, Dhont E, Peperstraete H, Delanghe JR, Vande Walle J, De Paepe P, De Cock PA. Augmented renal clearance: a common condition in critically ill children. Pediatr Nephrol. 2019; 34: 1099-

1106.https://www.ncbi.nlm.nih.gov/pubmed/30778827

[29] Kwatra NS, Meany HJ, Ghelani SJ, Zahavi D, Pandya N, Majd M. Glomerular hyperfiltration in children with cancer: prevalence and a hypothesis. Pediatr Radiol. 2017; 47: 221-

226.https://www.ncbi.nlm.nih.gov/pubmed/27812743

[30] Hjorth L, Wiebe T, Karpman D. Hyperfiltration evaluated by glomerular filtration rate at diagnosis in children with cancer. Pediatr Blood Cancer. 2011; 56: 762-766.https://www.ncbi.nlm.nih.gov/pubmed/21370408

[31] Hirai K, Ihara S, Kinae A, Ikegaya K, Suzuki M, Hirano K, Itoh K. Augmented Renal Clearance in Pediatric Patients With Febrile Neutropenia Associated With Vancomycin Clearance. Ther Drug Monit. 2016; 38: 393-397.https://www.ncbi.nlm.nih.gov/pubmed/27172381

[32] Bialkowski S, Staatz CE, Clark J, Lawson R, Hennig S. Gentamicin Pharmacokinetics and Monitoring in Pediatric Patients with Febrile Neutropenia. Ther. Drug Monit. 2016; 38: 693-

698.http://www.ncbi.nlm.nih.gov/pubmed/27851686

[33] Kim HS, Park BK, Kim SK, Han SB, Lee JW, Lee DG, Chung NG, Cho B, Jeong DC, Kang JH. Clinical characteristics and outcomes of Pseudomonas aeruginosa bacteremia in febrile neutropenic children and adolescents with the impact of antibiotic resistance: a retrospective study. BMC Infect Dis. 2017; 17 : 500.https://www.ncbi.nlm.nih.gov/pubmed/28716109

[34] van Donge T, Bielicki JA, van den Anker J, Pfister M. Key Components for Antibiotic Dose Optimization of Sepsis in Neonates and Infants. Front Pediatr. 2018; 6:

325.https://www.ncbi.nlm.nih.gov/pubmed/30420947 
780 amikacin TDM results (392 trough, 388 peak)

302 treatment episodes

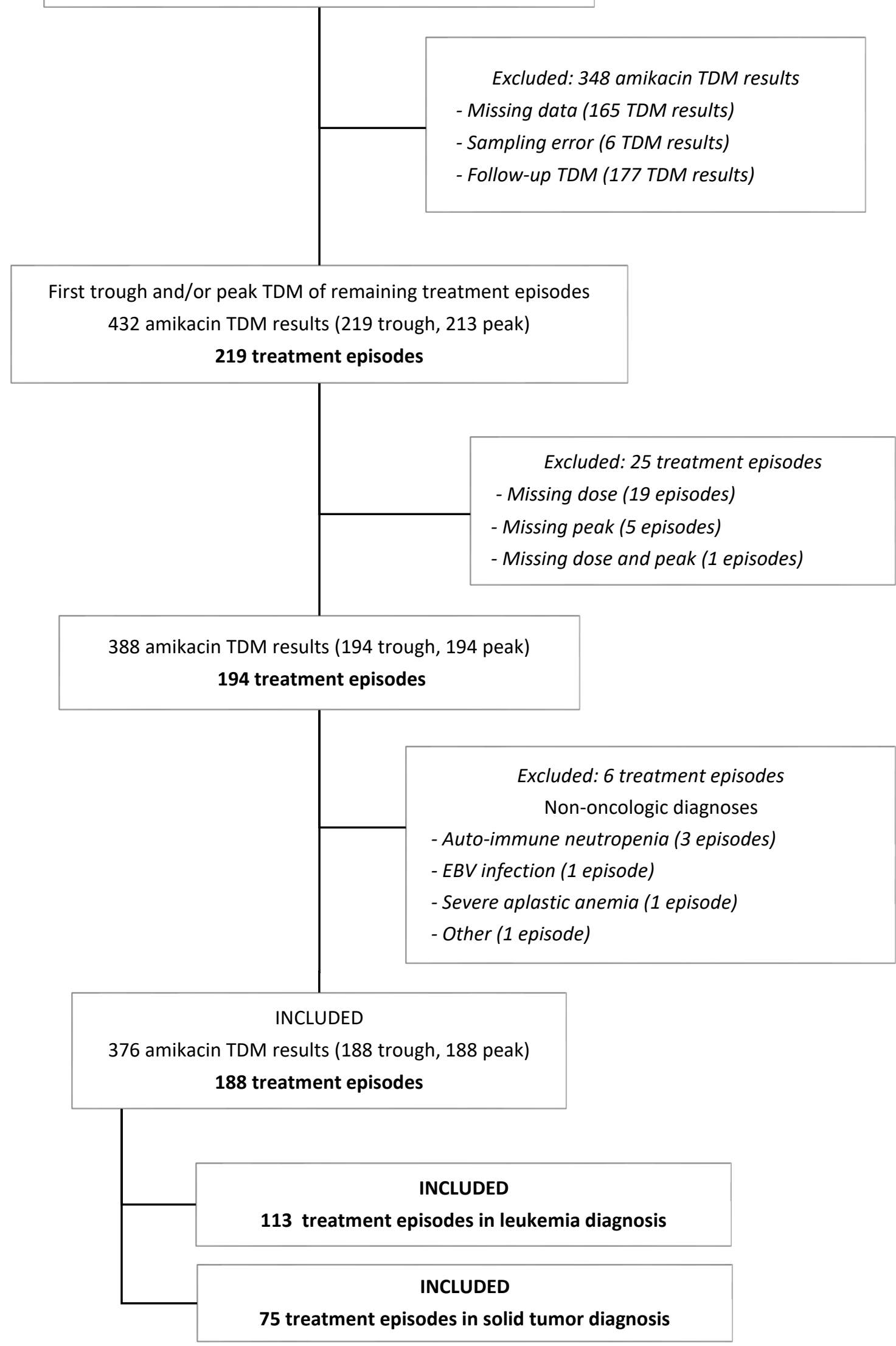

Figure 1 
Figure 2: The nonlinear trend (+ 95\% confidence interval in dashed lines) for amikacin clearance $(\mathrm{Cl}, \mathrm{L} / \mathrm{h})$ by height, for median creatinine of the entire dataset, is presented in Figure 2a. The linear trend (+95\% CI in dashed lines) for $\mathrm{Cl}$ by creatinine, for median height of the entire dataset, is presented in Figure 2b. In the multivariable model, $\mathrm{Cl}$ is expressed as $\mathrm{Cl}(\mathrm{L} / \mathrm{h})$ $=1.1968-0.0179 *$ height $+0.0002 *$ height $*$ height $-1.1032 *$ creatinine $($ with height in $\mathrm{cm}$, creatinine in $\mathrm{mg} / \mathrm{dL}$ ).

\section{Figure 2a}

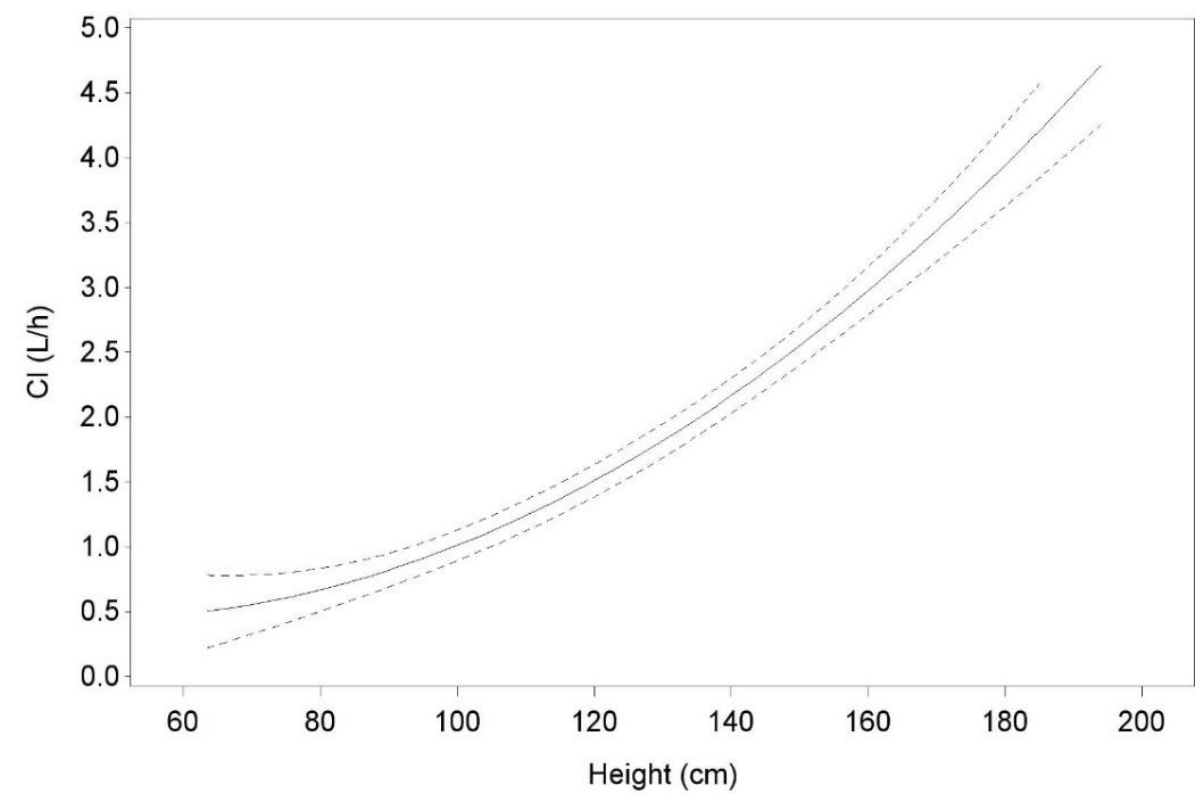

Figure 2b

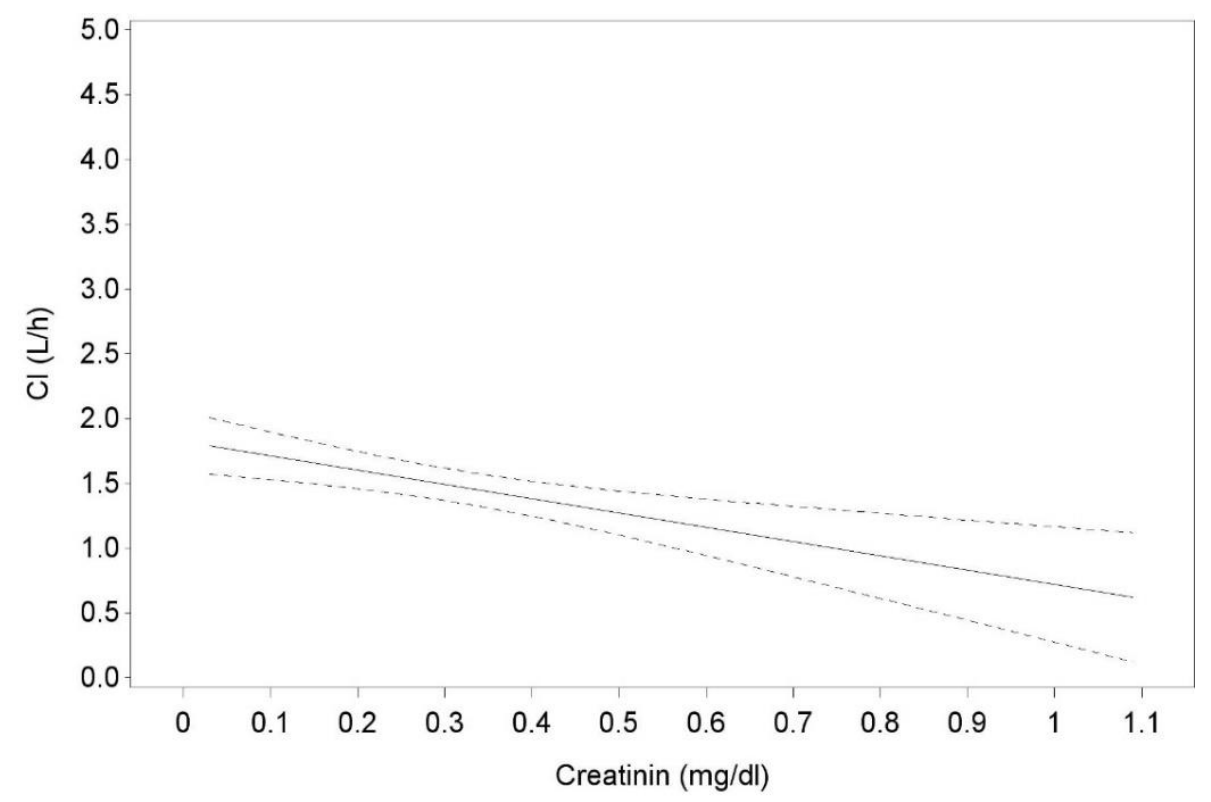


Figure 3: Graphs presenting the linear trends for volume of distribution (Vd, L) by height, for median creatinine and weight (Figure 3a), and by creatinine, for median height and weight (Figure $3 b$ ). Medians are calculated based on the entire dataset. In the multivariable model, Vd is expressed as $\mathrm{Vd}(\mathrm{L})=-3.5156+0.0936 *$ weight $+0.1041 *$ height $-7.0313 *$ creatinine (with weight in $\mathrm{kg}$, height in $\mathrm{cm}$, creatinine in $\mathrm{mg} / \mathrm{dL}$ ).

\section{Figure 3a}

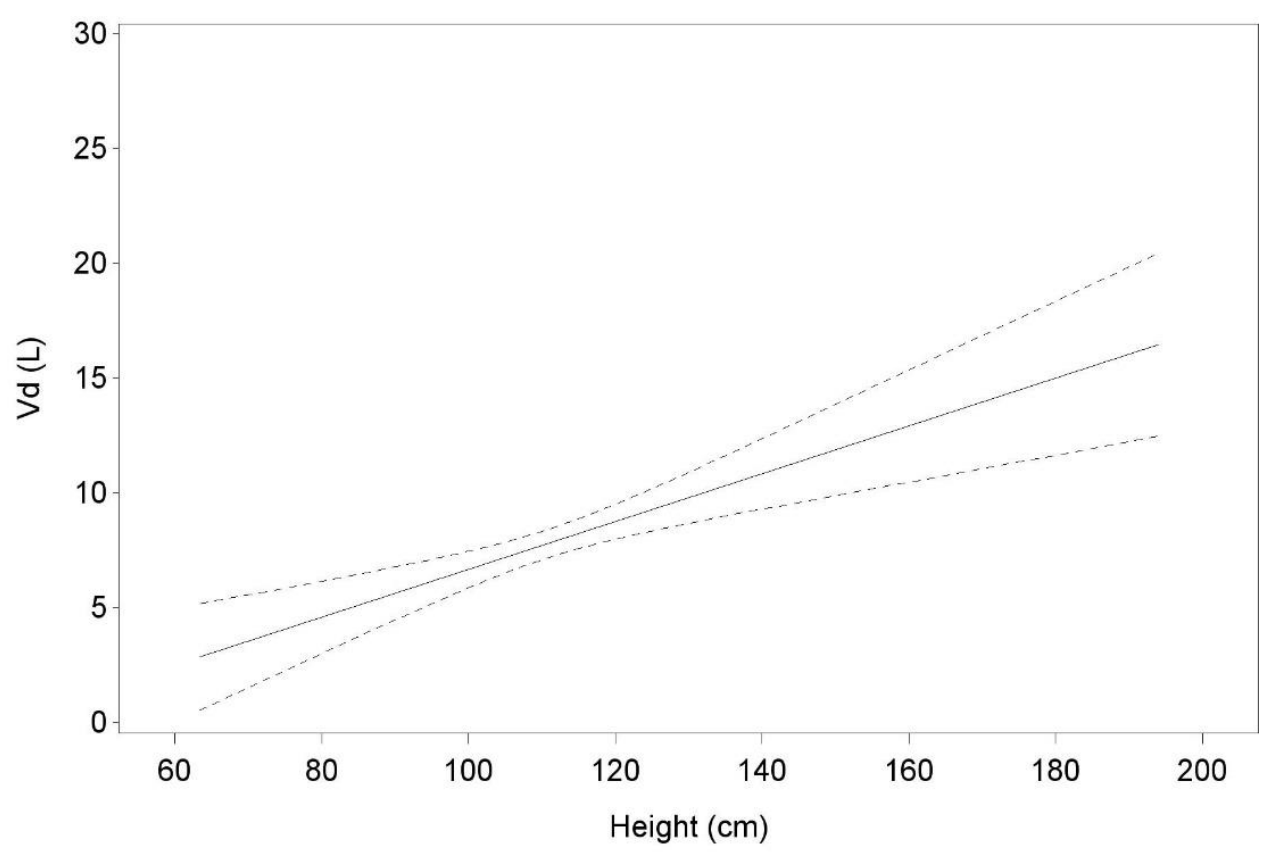

Figure 3b

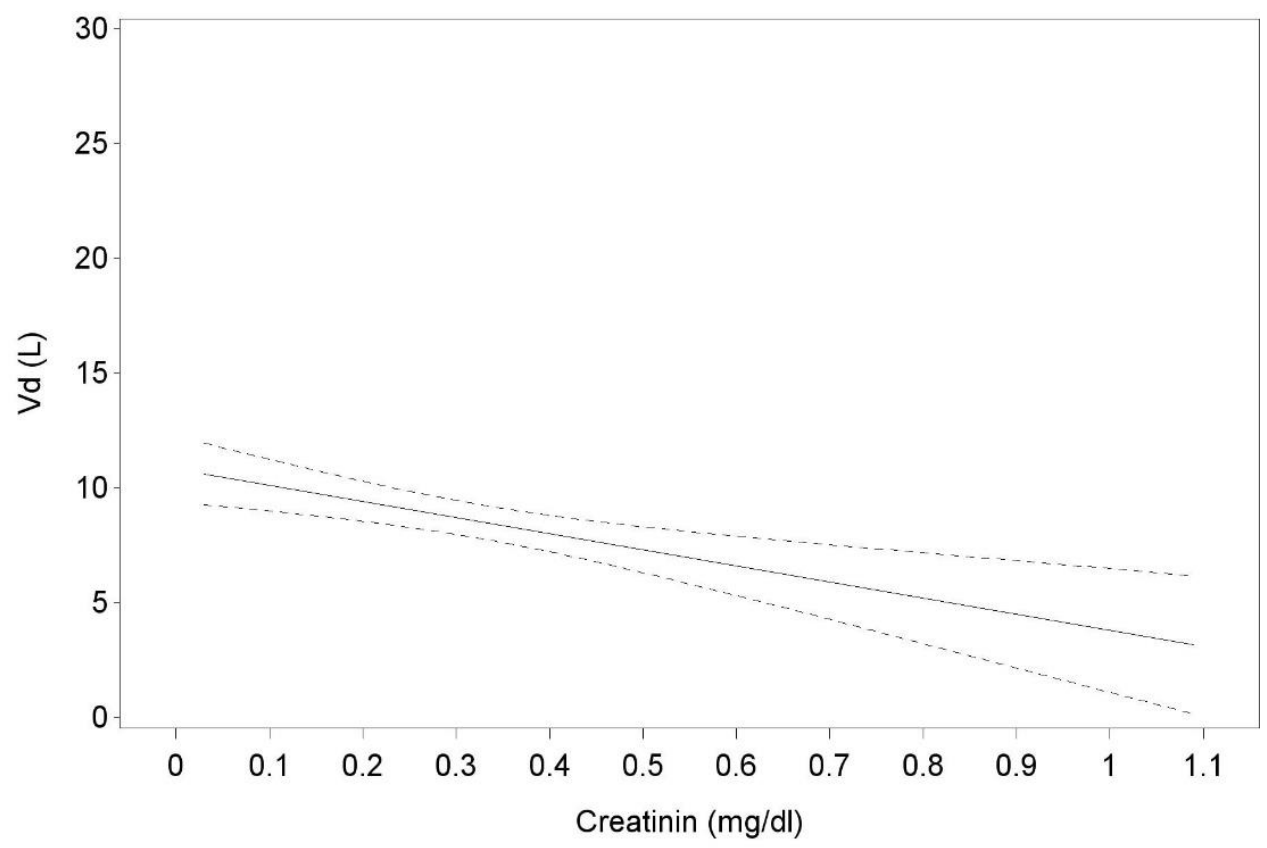


Table 1: Clinical and biochemical characteristics of the included observations.

\begin{tabular}{|c|c|c|}
\hline Continuous characteristic & Median & IQR \\
\hline Age (months) & 80.00 & $40.50 ; 151.50$ \\
\hline Height $(\mathrm{cm})$ & 118.75 & $100.00 ; 156.65$ \\
\hline Weight (kg) & 21.15 & $14.30 ; 45.45$ \\
\hline Creatinemia $(\mathrm{mg} / \mathrm{dL})^{a}$ & 0.31 & $0.24 ; 0.49$ \\
\hline Albuminemia $(\mathrm{g} / \mathrm{L})^{\mathrm{b}}$ & 36.70 & $33.00 ; 39.30$ \\
\hline CRP (mg/L) & 73.80 & $33.25 ; 149.15$ \\
\hline Amikacin dose $(\mathrm{mg} / \mathrm{kg})$ & 20.00 & $19.45 ; 20.52$ \\
\hline Dichotomous characteristic & Incidence & Percentage (\%) \\
\hline $\begin{array}{ll}\text { Sex } & \text { (male) } \\
& \text { (female) }\end{array}$ & $\begin{array}{l}100 / 188 \\
88 / 188\end{array}$ & $\begin{array}{l}53.19 \\
46.81\end{array}$ \\
\hline $\begin{array}{ll}\text { Diagnosis } & \text { (leukemia) } \\
& \text { (solid tumor) }\end{array}$ & $\begin{array}{l}113 / 188 \\
75 / 188\end{array}$ & $\begin{array}{l}60.11 \\
39.89\end{array}$ \\
\hline $\begin{array}{r}\text { Induction phase (yes) } \\
\text { (no) }\end{array}$ & $\begin{array}{l}23 / 188 \\
165 / 188\end{array}$ & $\begin{array}{l}12.23 \\
87.77\end{array}$ \\
\hline $\begin{array}{ll}\text { Cisplatinum } & \text { (yes) } \\
& (\text { no })\end{array}$ & $\begin{array}{l}7 / 188 \\
181 / 188\end{array}$ & $\begin{array}{l}3.72 \\
96.28\end{array}$ \\
\hline $\begin{array}{cc}\text { Carboplatinum } & \text { (yes) } \\
& (\text { no })\end{array}$ & $\begin{array}{l}15 / 188 \\
173 / 188\end{array}$ & $\begin{array}{l}7.98 \\
92.02\end{array}$ \\
\hline $\begin{array}{ll}\text { Ifosfsamide } & \text { (yes) } \\
& (\text { no })\end{array}$ & $\begin{array}{l}46 / 188 \\
142 / 188\end{array}$ & $\begin{array}{l}24.47 \\
75.53\end{array}$ \\
\hline $\begin{aligned} \text { Blood culture }^{\mathrm{c}} & \text { (positive) } \\
& \text { (negative) }\end{aligned}$ & $\begin{array}{l}25 / 178 \\
153 / 178\end{array}$ & $\begin{array}{l}14.04 \\
85.96\end{array}$ \\
\hline
\end{tabular}

${ }^{\mathrm{a}}$ creatinemia available in 187/188 cases; ${ }^{\mathrm{b}}$ albuminemia available in 182/188 cases; ${ }^{\mathrm{c}}$ blood culture data unknown in 10/188 cases. CRP: C-reactive protein; IQR: interquartile range. 
Table 2: Univariable analyses of (non-)maturational covariates of clearance $(\mathrm{Cl}, \mathrm{L} / \mathrm{h})$

\begin{tabular}{|c|c|c|c|c|}
\hline Covariate & Test & Estimate (95\% CI) & P-value & Nobs. \\
\hline Age & +1 month & $0.01354(0.01194 ; 0.01514)$ & $<0.0001 *$ & 188 \\
\hline Weight & $+1 \mathrm{~kg}$ & $0.04685(0.04172 ; 0.05199)$ & $<0.0001^{*}$ & 188 \\
\hline Height & $+1 \mathrm{~cm}$ & $0.02613(0.02335 ; 0.02892)$ & $<0.0001 *$ & 188 \\
\hline Height & Quadratic trend & & $0.0003 *$ & 188 \\
\hline Albuminemia & $+1 \mathrm{~g} / 1$ & $-0.0006(-0.0274 ; 0.02609)$ & 0.9626 & 182 \\
\hline Creatinemia & $+0.1 \mathrm{mg} / \mathrm{dl}$ & $0.17576(0.10310 ; 0.24842)$ & $<0.0001 *$ & 187 \\
\hline Creatinemia & Quadratic trend & & $0.0105 *$ & 187 \\
\hline CRP & $+1 \mathrm{mg} / \mathrm{l}$ & $0.00150(0.00014 ; 0.00286)$ & $0.0306^{*}$ & 188 \\
\hline CRP & Quadratic trend & & $0.0130 *$ & 188 \\
\hline Carboplatinum & Yes vs no & $-0.0117(-0.6035 ; 0.58018)$ & 0.9688 & 188 \\
\hline Cisplatinum & Yes vs no & $-0.4083(-1.340 ; 0.52303)$ & 0.3852 & 188 \\
\hline Diagnose & Solid tumor vs leukemia & $0.32424(-0.0551 ; 0.70358)$ & 0.0927 & 188 \\
\hline Sex & Female vs male & $-0.2123(-0.5893 ; 0.16470)$ & 0.2655 & 188 \\
\hline Blood culture & Positive vs negative & $-0.2217(-0.6157 ; 0.17223)$ & 0.2654 & 178 \\
\hline Ifosfamide & Yes vs no & $0.32358(-0.1146 ; 0.76180)$ & 0.1454 & 188 \\
\hline Induction phase & Yes vs no & $0.00393(-0.3779 ; 0.38576)$ & 0.9837 & 188 \\
\hline
\end{tabular}

CI: confidence interval; CRP: C-reactive protein. For continuous covariates the estimate provided is the slope (change in outcome for 1-unit increase in predictor), for dichotomous covariates the estimate provided is the difference $[>(<) 0$ means higher (lower) outcome level in the first category]. * $\mathrm{P}$-values $<0.05$ were considered significant. 
Table 3a: Table 3a presents the final multivariable model for clearance $(\mathrm{Cl}, \mathrm{L} / \mathrm{h})$ resulting from a backward selection procedure; i.e. the selected covariates with their parameter estimates and significance.

\begin{tabular}{|l|l|l|l|l|}
\hline Covariates & $\begin{array}{l}\text { Parameter } \\
\text { estimate }\end{array}$ & SE & P-value & N obs. \\
\hline Intercept & 1.1968 & 0.6129 & 0.0533 & 187 \\
\hline Height & -0.0179 & 0.01017 & 0.0827 &. \\
\hline Height*Height & 0.0002 & 0.000041 & $<0.0001$ &. \\
\hline Creatinemia & -1.1032 & 0.3132 & 0.0008 &. \\
\hline
\end{tabular}

SE: standard error.

Table 3b: Table $3 \mathrm{~b}$ presents the covariate effects with $95 \%$ confidence intervals. Given that height follows a nonlinear trend, its effect on outcome is not constant over time, hence the effect is estimated at 3 different heights (i.e. percentile p25-50-75 of the study population, presented as rounded values $100-120-150 \mathrm{~cm}$ ).

\begin{tabular}{|l|l|l|l|}
\hline Covariate & Test & Effect estimate (95\% CI) & P-value \\
\hline Height $100 \mathrm{~cm}$ & $+1 \mathrm{~cm}$ & $0.0212(0.0163 ; 0.0261)$ & $<0.0001$ \\
\hline Height $120 \mathrm{~cm}$ & $+1 \mathrm{~cm}$ & $0.0290(0.0255 ; 0.0326)$ & $<0.0001$ \\
\hline Height $150 \mathrm{~cm}$ & $+1 \mathrm{~cm}$ & $0.0407(0.0347 ; 0.0467)$ & $<0.0001$ \\
\hline Creatinemia & $+0.1 \mathrm{mg} / \mathrm{dL}$ & $-0.1103(-0.1728 ;-0.0479)$ & 0.0008 \\
\hline
\end{tabular}

CI: confidence interval. The effect estimate is the change in CL $(\mathrm{L} / \mathrm{h})$ for the indicated increase of the predictor level. 
Table 4: Univariable analyses of (non-)maturational covariates of volume of distribution (Vd, L).

\begin{tabular}{|c|c|c|c|c|}
\hline Covariate & Test & Estimate (95\% CI) & P-value & N obs. \\
\hline Age & +1 month & $0.06272(0.05283 ; 0.07261)$ & $<0.0001 *$ & 188 \\
\hline Weight & $+1 \mathrm{~kg}$ & $0.21191(0.17830 ; 0.24552)$ & $<0.0001 *$ & 188 \\
\hline Weight & Quadratic trend & & $0.0487^{*}$ & 188 \\
\hline Height & $+1 \mathrm{~cm}$ & $0.12316(0.10574 ; 0.14059)$ & $<0.0001 *$ & 188 \\
\hline Albumin & $+1 \mathrm{~g} / \mathrm{l}$ & $-0.0378(-0.1771 ; 0.10155)$ & 0.5902 & 182 \\
\hline Creatinemia & $+0.1 \mathrm{mg} / \mathrm{dl}$ & $0.59499(0.20376 ; 0.98621)$ & $0.0034 *$ & 187 \\
\hline Creatinemia & Quadratic trend & & $0.0207 *$ & 187 \\
\hline CRP & $+1 \mathrm{mg} / \mathrm{l}$ & $0.00706(0.00000 ; 0.01413)$ & $0.0499 *$ & 188 \\
\hline CRP & Quadratic trend & & $0.0451 *$ & 188 \\
\hline Carboplatinum & Yes vs no & $-0.2230(-3.325 ; 2.8793)$ & 0.8865 & 188 \\
\hline Cisplatinum & Yes vs no & $-2.316(-7.214 ; 2.5829)$ & 0.3493 & 188 \\
\hline Diagnose & Solid tumor vs leukemia & $0.93094(-1.083 ; 2.9452)$ & 0.3601 & 188 \\
\hline Gender & Female vs male & $-1.197(-3.179 ; .78520)$ & 0.2327 & 188 \\
\hline Blood culture & Positive vs negative & $-1.541(-3.587 ; .50453)$ & 0.1374 & 178 \\
\hline Ifosfamide & Yes vs no & $1.8253(-.4768 ; 4.1273)$ & 0.1184 & 188 \\
\hline Induction phase & Yes vs no & $0.20673(-1.774 ; 2.1871)$ & 0.8358 & 188 \\
\hline
\end{tabular}

CI: confidence interval; CRP: C-reactive protein. For continuous covariates the estimate provided is the slope (change in outcome for 1-unit increase in predictor), for dichotomous covariates the estimate provided is the difference $[>(<) 0$ means higher (lower) outcome level in the first category]. $*$ P-values $<0.05$ were considered significant. 
Table 5: The final multivariable model for volume of distribution $(\mathrm{Vd}, \mathrm{L})$ resulting from a backward selection procedure; i.e. the selected covariates with their parameter estimates and significance as well as the covariate effects with $95 \%$ confidence intervals.

\begin{tabular}{|c|c|c|c|c|c|c|}
\hline Covariate & Parameter estimate & SE & Test & Effect estimate (95\% CI) & P-value & N obs. \\
\hline Intercept & -3.5156 & 1.8479 & & & 0.0597 & 187 \\
\hline Weight & 0.0936 & 0.04336 & $+1 \mathrm{~kg}$ & $0.0936(0.0071 ; 0.1800)$ & 0.0344 & . \\
\hline Height & 0.1041 & 0.02368 & $+1 \mathrm{~cm}$ & $0.1041(0.0569 ; 0.1513)$ & $<.0001$ & . \\
\hline Creatinemia & -7.0313 & 1.9118 & $+0.1 \mathrm{mg} / \mathrm{dl}$ & $-.7031(-1.084 ;-.3218)$ & 0.0005 & . \\
\hline
\end{tabular}

SE: standard error. The effect estimate is the change in $\mathrm{Vd}(\mathrm{L})$ for the indicated increase of the predictor level. 
Table S1: The multivariable model for clearance $(\mathrm{Cl}, \mathrm{L} / \mathrm{h} . \mathrm{BSA})$ resulting from a backward selection procedure; i.e. the selected covariates with their parameter estimates and significance as well as the covariate effects with $95 \%$ confidence intervals.

\begin{tabular}{|c|c|c|c|c|c|c|}
\hline Covariate & $\begin{array}{l}\text { Parameter } \\
\text { estimate }\end{array}$ & SE & Test & Effect estimate $(95 \%$ CI) & P-value & N obs. \\
\hline Intercept & 1.2241 & 0.1656 & & & $<0.0001$ & 187 \\
\hline Height & 0.0075 & 0.0017 & $+1 \mathrm{~cm}$ & $0.0075(0.0041 ; 0.0109)$ & $<0.0001$ & . \\
\hline Creatinemia & -1.0520 & 0.2836 & $+0.1 \mathrm{mg} / \mathrm{dL}$ & $-0.1052(-.1618 ;-0.0486)$ & 0.0004 & . \\
\hline
\end{tabular}

SE: standard error. Predicted $\mathrm{Cl}$ (L/h.BSA) is the weighted sum of covariate measurements, with parameter estimates as weights

The effect estimate is the change in $\mathrm{Cl}$ (L/h.BSA) for indicated increase of the predictor level. A p-value $<0.05$ is considered statistically significant. 
Table S2: a) presents the multivariable model for clearance $(\mathrm{Cl}, \mathrm{L} / \mathrm{kg} / \mathrm{h})$ resulting from a backward selection procedure; i.e. the selected covariates with their parameter estimates and significance. b) Presents the covariate effects with $95 \%$ confidence intervals. Given that creatinemia follows a nonlinear trend, its effect on outcome is not constant over time, hence the effect is estimated at 3 different creatinine values (i.e. percentile p25-5075 of the study population, presented as rounded values $0.3-0.5-0.7 \mathrm{mg} / \mathrm{dL}$ ).

a)

\begin{tabular}{|l|l|l|l|}
\hline Covariate & Parameter estimate & SE & P-value obs. \\
\hline Intercept & 0.0991 & 0.0065 & $<0.0001$ \\
\hline Creatinemia & -0.1228 & 0.0296 & $<0.0001$ \\
\hline Creatinemia*Creatinemia & 0.0748 & 0.0288 & 0.0114 \\
\hline
\end{tabular}

SE: standard error. Predicted CL ( $\mathrm{L} / \mathrm{kg} / \mathrm{h})$ is the weighted sum of variable measurements, with parameter estimates as weights. A P-value <0.05 is considered statistically significant.

b)

\begin{tabular}{|l|l|l|l|}
\hline Covariate & test & Effect estimate (95\% CI) \\
\hline Creatinemia $0.3 \mathrm{mg} / \mathrm{dL}$ & $+0.1 \mathrm{mg} / \mathrm{dL}$ & $-0.0070(-0.0094 ;-0.0047)$ \\
\hline Creatinemia $0.5 \mathrm{mg} / \mathrm{dL}$ & $+0.1 \mathrm{mg} / \mathrm{dL}$ & $-0.0041(-0.0058 ;-0.0023)$ & $<0.0001$ \\
\hline Creatinemia $0.7 \mathrm{mg} / \mathrm{dL}$ & $+0.1 \mathrm{mg} / \mathrm{dL}$ & $-0.0011(-0.0044 ; 0.0023)$ & 0.5275 \\
\hline
\end{tabular}


CI: confidence interval. The effect estimate is the change in CL ( $\mathrm{L} / \mathrm{kg} / \mathrm{h})$ for indicated increase of the predictor level. Creatinemia: due to the quadratic effect, the change in CL

$(\mathrm{L} / \mathrm{kg} / \mathrm{h})$ for a $0.1 \mathrm{mg} / \mathrm{dL}$ increase varies over the range of creatinimia. e.g. the change in $\mathrm{CL}(\mathrm{L} / \mathrm{kg} / \mathrm{h})$ is different when creatinine goes from $0.3 \mathrm{mg} / \mathrm{dL}$ to $0.4 \mathrm{mg} / \mathrm{dL}$ compared to

$0.5 \mathrm{mg} / \mathrm{dL}$ to $0.6 \mathrm{mg} / \mathrm{dL}$. A P-value $<0.05$ is considered statistically significant. 